\title{
MODELLING OF A VIBRATION-DRIVEN MODULE FOR CAPSULE LOCOMOTION SYSTEMS
}

\author{
KHAC-TUAN NGUYEN ${ }^{1}$, VAN-DU NGUYEN ${ }^{2}$, KY-THANH HO ${ }^{3}$ \& NGOC-TUAN LA ${ }^{4}$ \\ ${ }^{1,2,3}$ Research Scholar, Thai Nguyen University of Technology, Thai Nguyen University, Vietnam \\ ${ }^{4}$ Research Scholar, Vinh University of Technology Education, Vietnam
}

\begin{abstract}
This paper presents a model for locomotion systems based on the combination of vibration and impact of an oscillating mass with two-side elastic constraints. The proposed system is excited by a periodic half-sine waveform force. A mathematical model was developed to form a basis of the design and to choose proper operational parameters. The numerical solution showed that the system was able to move backward and forward as desired by simply reversing the excitation force. Several advantages of the new model, compared to the recent platform excited by square force were highlighted. Dynamics response of the new model was also analyzed by applying bifurcation technique. In addition, the new model was proved to able to move in desired direction under both anisotropic and isotropic friction conditions. This model is promising to apply in capsule robots, where the whole system must be encapsulated in a smooth form and should be able to move in different friction conditions.
\end{abstract}

KEYWORDS: Self-Propulsion; Vibration-Driven Locomotion; Capsuled Robot; Isotropic Friction \& Anisotropic Friction

Received: Apr 21, 2020; Accepted: May 12, 2020; Published: May 29, 2020; Paper Id.: IJMPERDJUN202075

\section{INTRODUCTION}

There have been growing demands and remarkable development of mobile systems witnessed in recent years, especially in the field of locomotion robots. The systems have extensive prospective applications, ranged from large-size systems (Cazalilla, Vallés, Valera, Mata, \& Díaz-Rodríguez, 2016) working in remote and hostile environments, disaster rescues, pipeline inspection...(Yaguchi, Kamata, \& Sugawara, 2016), (Shukla \& Karki, 2016) to miniature robots for medical assistance (Mohamed, Elgamal, \& Elsharkawy, 2018). Conventional mobile systems usually consist of external actuation components, such as wheels, legs or paddles, which would have restrictions in mechanical complexity, physical size, failure of moving parts, and affecting hazard to working environment. Consequently, the design of new locomotion systems without external driving mechanism is required so that it can be easily encapsulated in a smooth form. There have been two design principles of such encapsulated locomotion systems, as briefly summarized below.

The two-mass system, initially proposed by Chernousko in 2002 (Chernous'ko, 2002), provides a promising propulsion mechanism for expected limbless locomotion platforms. In this system, the rectilinear motion of the primary mass (the system body) can be achieved by using a periodically driven internal mass interacting with the main body in the presence of dry friction. The internal mass must be controlled to move inside with special multi-phase accelerations (see (Huda \& Yu, 2015), (Nikolay Bolotnik, 2015), (Su, Zhang, Tan, \& Li, 2009), (Li, Furuta, \&Chernousko, 2006) for example). The locomotion can be generated when the inertial force, caused by acceleration changes, exceeds the friction threshold between the body and the surrounding environment. The major 
advantage of this two-mass system is its structural simplicity and it would be perfect to form capsuled robots. In addition, by reversing the excitation pole, the movement direction of the system would also be inverted. It would be worth noting that for capsule endoscopy applications, the system abilities for moving forwards and backwards are required (Y. Liu, Pavlovskaia, Wiercigroch, \& Peng, 2015). Besides, the two-mass systems still has a shortcoming of demanding a complex motion control, leading to difficulties in setting-up and arranging the control hardware inside the robot. To date, several other studies have tried to simplify the control algorithm by using sinusoidal excitation (Xu \& Fang, 2019), (Du, Fang, Zhan, \& Xu, 2018), (Zhan, Xu, \& Fang, 2018). However, such systems can only move in desired direction in a special case of anisotropic friction, where the friction for the preferred motion is less than that for opposite motion. This condition of friction would not be always available in practice.

Another choice of design for self-propelled systems is employing vibrational actuators where the interaction of the vibration combined with impacts between an internal mass and the system body. In these systems, the internal mass oscillates and periodically collides with the body. This results in a jump-up of the inertial force. When the interaction force exceeds the friction threshold, the system will move in the direction of the impact force. The drifting oscillator was initially proposed by Pavlovskaia et al. in 2003 (E. Pavlovskaia, Wiercigroch, \& Grebogi, 2001), provided a basis for developing distinctive locomotion systems. The internal mass (impact oscillator) can be driven by several ways, for example by a system of motor and cam mechanism (Ekaterina Pavlovskaia, Wiercigroch, Woo, \& Rodger, 2003), by solenoids working with sinusoidal in an RLC circuit (V.-D. Nguyen, Woo, \& Pavlovskaia, 2008), (V. D. Nguyen \& Woo, 2008), by electromagnetic devices (Grankin \& Yatsun, 2009), (Sapronov, Cherepanov, \& Yatsun, 2010), by a linear motor (Y. Liu, Pavlovskaia, Wiercigroch, et al., 2015), or by electro-dynamic shakers (V.-D. Nguyen, Ho, Duong, Chu, \& Ngo, 2017). The vibration-driven platforms have been extensively investigated from various aspects, such as designing (Yan, Liu, Manfredi, \& Prasad, 2019), (Duong et al., 2018); modeling(Y. Liu, Wiercigroch, Pavlovskaia, \& Yu, 2013), (Yan, Liu, Páez Chávez, Zonta, \& Yusupov, 2018), (Du et al., 2018), (V.-D. Nguyen, Nguyen, Ngo, \& La, 2017); experimental validation (Y. Liu, Pavlovskaia, Wiercigroch, et al., 2015), (Sobolev \& Sorokin, 2007); dynamical analysis (P. Liu, Yu, \& Cang, 2019), (Nunuparov, Becker, Bolotnik, Zeidis, \& Zimmermann, 2019), (Zhan et al., 2018), (V.-D. Nguyen, Ho, et al., 2017), (V.-D. Nguyen, Duong, Chu, \& Ngo, 2017); optimal progression (Fan et al., 2020), (Nunuparov et al., 2019), (Xu \& Fang, 2019), (P. Liu, Yu, \& Cang, 2018), (Golitsyna, 2018); and motion control (Y. Liu, Pavlovskaia, Wiercigroch, et al., 2015), (V.-D. Nguyen, Ho, et al., 2017),(Chernousko, 2018), etc. In order to provide the abilities for moving forwards and backwards, a position feedback control method for the vibro-impact capsule system was proposed (Y. Liu, Pavlovskaia, Wiercigroch, et al., 2015). By changing the control gain from negative to positive, the direction of motion can change from backward to forward. However, the desired direction of the system motion as well as the motion itself can only be obtained by carefully choosing proper parameters. To address this issue, a 2-side piecewise-linear impact oscillator working with periodic excitation was recently proposed (Duong et al., 2018). Such system contains two-side symmetric constrains and was excited by an impulse periodic force (in square-wave form). The backward and forward motions were easily achieved by applying the force in the desired direction. However, the major drawback of that design is that in practice, the non-ideal power source is not able to change exactly in the square form. In addition, the system still has a rich dynamical response, from periodic motions to chaotic. Hence, this paper presents a new development for locomotion system with a hope to address the issues mentioned. In the new model, the excitation force was suggested in the form of a half-sine wave, which can be easily obtained from any common sinusoidal power source by means of an electrical rectifier. Also, the proposed system remains the ability of changing the motion direction by exchanging the direction of the force. In addition, the 
working ability and dynamic response of the new model in both conditions of isotropic and anisotropic friction forces was also evaluated. For the best of the authors' knowledge, it has rarely been found studies implementing such evaluation.

The paper is organized as follows. Basic mechanics and the mathematical model of the new proposed system will be depicted in Section 2. Section 3 presents the performance, dynamic response and major advantages of the new system compared to a similar recent system. Finally, conclusions are given in Section 4.

\section{BASIC MECHANICS OF THE VIBRATION-DRIVEN LOCOMOTION ACTUATOR}

Figure 1 presents two recently developed models, a one-side impact (figure 1a) and a two-side impact (figure 1b) for vibration-driven locomotion systems. Each model consists of a body mass, $m_{2}$ and an internal mass $m_{1}$, connecting together by means of an elastic spring with stiffness $k_{1}$ and a viscous damper with damping coefficient $c$. On each model, $X_{1}$ and $X_{2}$ represent the absolute displacements of the internal mass and the body mass, respectively. The internal mass $m_{l}$ oscillates and periodically collides with the body $m_{2}$ when the relative displacement is larger than or equals to the gap G. The motion of the system takes place when the force acting on the body from the active mass exceeds the threshold of the dry friction force $F_{f}$ between the body and the environment surface.

The one-side model (See figure 1a) has been considered in abundant studies (See for example (Yan et al., 2019), (V.-D. Nguyen, Duong, et al., 2017), (Gu \& Deng, 2018), (Y. Liu, Islam, Pavlovskaia, \& Wiercigroch, 2016), (Y. Liu, Pavlovskaia, Hendry, \& Wiercigroch, 2013). The internal mass was usually driven by a sinusoidal force. In order to achieve a desired direction (backward or forward motion), it is required to carefully choosing proper values of several parameters, by applying a position feedback control law to the system with the Coulomb friction model (Y. Liu, Pavlovskaia, Wiercigroch, et al., 2015).

(a)

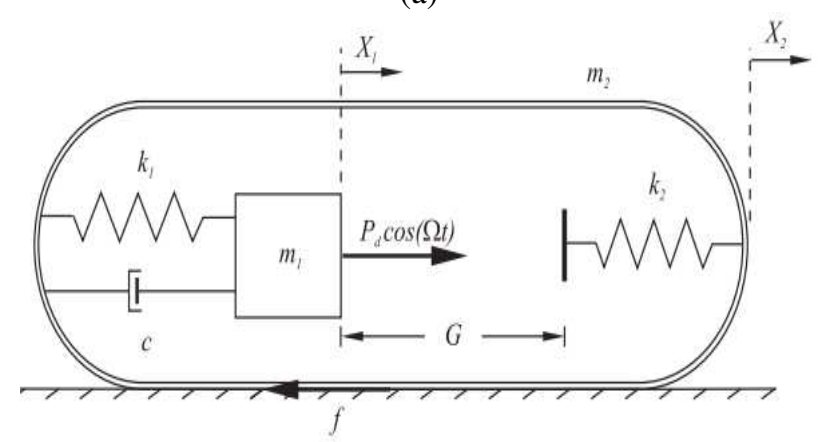

(b)

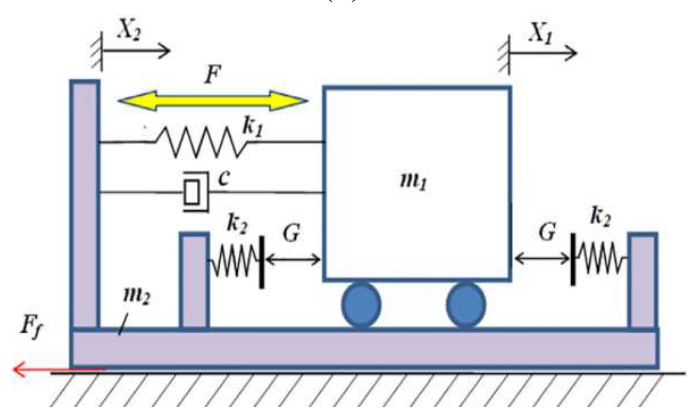

Figure 1: Recent Locomotion Systems: (a) One-Side Impact (Y. Liu, Wiercigroch, et al., 2013) and (b) Two-Side Impact (Duong et al., 2018).

In the two-side impact system (Duong et al., 2018), the desired backward or forward motion can be achieved by simply applying a periodic square force in the same direction. Better performance and stable dynamic response can be found by controlling proper parameters, such as amplitude and frequency of the excitation force. The major concern of this model is that it would be difficult to generate exactly the rectangular form of the impulse force in practice.

The new proposed model is developed from the two-side model as shown in Figure $1 \mathrm{~b}$. A movable mass $m 1$ is connected with the system frame $m 2$ by a linear spring with stiffness $k 1$ and a viscous damper with damping coefficient $c$. Two rigid obstacle blocks are symmetrically located on both sides of the mass $m 1$ with a preset distance $G$. The impact 
stiffness of the obstacle blocks is simply modelled by a linear spring with stiffness $k_{2}$. The movement to the right side of the system is considered as forward direction. The inertial mass $m 1$ is driven by a force $F$ which is generated by either a linear motor, a pneumatic actuator or an electro-dynamic shaker. Hence, the force $F$ is modelled to be an interaction force between the two masses. In the proposed model, the force $F$ is in a half-sine form with amplitude $A$ and frequency $\Omega$. The amplitude, $A$ is positive if the force acts on the mass $m 1$ in the forward direction and vice versa. The friction force $F f$ has direction opposite to the velocity of the frame body. Suppose the excited force amplitude is positive, i.e. it acts on the in mass 1 in the forward direction. The mass 1 is driven and hit the right obstacle block, which would lead to the forward progression of the whole system. The reverse motion of the mass after impacting would be facilitated enough in duo by the reaction force and the restoring force from the spring $k 1$. It is supposed that the negative amplitude of the force is not necessary to drive the mass backward. As a result, the second half of a sinusoidal period should be omitted. Since the excitation force is zero and thus does not push the mass further to the left, the mass would not reach to the left obstacle block position. The excitation frequency must be controlled so as to have a harmonious relation with the natural motion of the impact mass.

It is to be noted that a half-sine power source can be easily obtained from any common sinusoidal source by means of an electrical rectifier. Figure 2 illustrates the excitation forces in the form of the squared wave (figure $2 a$ ) and the half-sine wave (figure $2 b$ ).

(a)

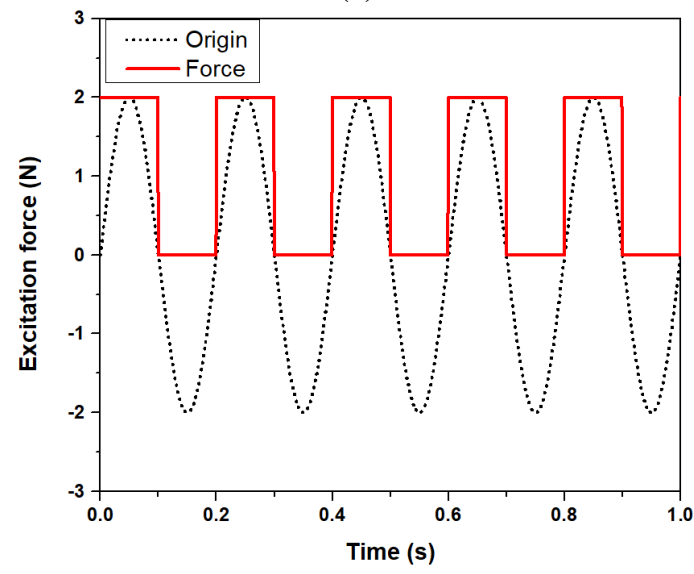

(b)

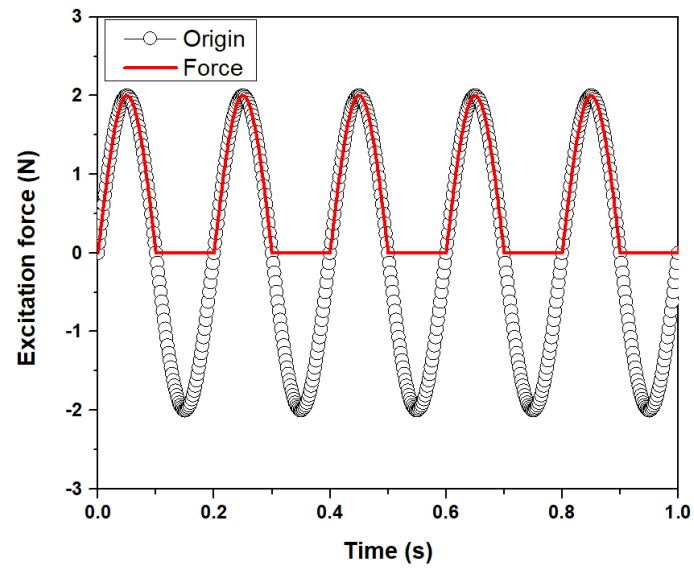

Figure 2: Excitation Force: (a) Square Form and (b) Half-Sine form.

As mentioned, an electrical power source in half-sine wave form can be easily transformed from a typical sine source by means of an electrical rectifier. Apply such power source in several types of electro-magnet devices such as a linear motor (such as in (Y. Liu, Pavlovskaia, \& Wiercigroch, 2015)), electro-magnet driver (Su et al., 2009) or electrodynamic shaker (V.-D. Nguyen, Duong, et al., 2017), (Duong et al., 2018), ones can easily obtain the half-sine excitation force.

The equations of motions of the two masses can be expressed as:

$$
\left\{\begin{array}{l}
m_{1} X_{1}^{\prime \prime}=F-k_{1} \cdot\left(X_{1}-X_{2}\right)-c \cdot\left(X_{1}^{\prime}-X_{2}^{\prime}\right)-\operatorname{sgn}\left(X_{1}-X_{2}\right) \cdot F_{i m p} \\
m_{2} X_{2}^{\prime \prime}=-F+k_{1} \cdot\left(X_{1}-X_{2}\right)+c \cdot\left(X_{1}^{\prime}-X_{2}^{\prime}\right)+\operatorname{sgn}\left(X_{1}-X_{2}\right) \cdot F_{i m p}-F_{r} \cdot \operatorname{sgn}\left(X_{2}^{\prime}\right)
\end{array}\right.
$$

where ()' and ()', denote the first and the second derivatives with respect to time; $F$ is the excitation force, which 
can be described as:

$$
F=\left\{\begin{array}{l}
A \sin (\Omega t), \sin (\Omega t)>0 \\
0, \sin (\Omega t) \leq 0
\end{array}\right.
$$

where $\Omega$ is the excitation frequency in $\mathrm{rad} / \mathrm{sec}, \Omega=2 . \pi f f, A$ and $f$ are the amplitude and frequency of the force, respectively. The impact force $F_{\text {imp }}$, generated when the mass $m_{l}$ hit the obstacle block, as similar in (Duong et al., 2018):

$$
F_{i m p}=\left\{\begin{array}{l}
k_{2} \cdot\left(\left|X_{1}-X_{2}\right|-G\right),\left(\left|X_{1}-X_{2}\right|-G\right)>0 \\
0,\left(\left|X_{1}-X_{2}\right|-G\right) \leq 0
\end{array}\right.
$$

Generally, the friction force can be modelled employing the Coulomb law, as following:

$$
F_{r}=\left\{\begin{array}{l}
R^{+}, X_{2}^{\prime}>0 \\
0, X_{2}^{\prime}=0 \\
R^{-}, X_{2}^{\prime}<0
\end{array}\right.
$$

where $\mathrm{R}^{+}$and $\mathrm{R}^{-}$are friction values for forward and backward motions, respectively.

In order to extend the results to different scales, Equations (1) were normalized by applying the following nondimensional variables and parameters:

$$
\begin{aligned}
& \tau=\Omega_{0} t ; x_{1}=\frac{k_{1}}{R^{-}} X_{1} ; x_{2}=\frac{k_{1}}{R^{-}} X_{2} ; x_{1}^{\prime}=\frac{d x_{1}}{d \tau} ; x_{2}^{\prime}=\frac{d x_{1}}{d \tau} ; \Omega_{0}=\sqrt{\frac{k_{1}}{m_{1}}} ; \omega=\frac{\Omega}{\Omega_{0}} \\
& \zeta=\frac{c}{2 m_{1} \Omega_{0}} ; \alpha=\frac{A}{R^{-}} ; \beta=\frac{k_{2}}{k_{1}} ; \gamma=\frac{k_{1}}{R^{-}} G ; \mu=\frac{m_{2}}{m_{1}} ; \rho=\frac{R^{+}}{R^{-}}
\end{aligned}
$$

The friction force thus can be expressed in dimensionless form as:

$$
f=\left\{\begin{array}{l}
\rho, x_{2}^{\prime}>0 \\
0, x_{2}^{\prime}=0 \\
-1, x_{2}^{\prime}<0
\end{array}\right.
$$

It is worth noting that, different situation of friction can be set by setting the value of $\rho$. For example, $\rho=1$ means to the condition of isotropic friction; $\rho<1$ describes one case of anisotropic frictional condition, where the friction force in forward motion is smaller than that in backward motion, and so on... In this study, $\rho$ will be treated as a parameter to evaluate its effect on the system behavior.

The final equations of motion for the whole system thus can be expressed as

$$
\left\{\begin{array}{l}
x_{1}^{\prime}=\alpha \cdot \sin (\omega \tau)-\left(x_{1}-x_{2}\right)-2 \zeta \cdot\left(x_{1}^{\prime}-x_{2}^{\prime}\right)-\operatorname{sgn}\left(x_{1}-x_{2}\right) \cdot H \\
x_{2}^{\prime}=\left[-\alpha \cdot \sin (\omega \tau)+\left(x_{1}-x_{2}\right)+2 \zeta \cdot\left(x_{1}^{\prime}-x_{2}^{\prime}\right)+\operatorname{sgn}\left(x_{1}-x_{2}\right) \cdot H-\rho \cdot \operatorname{sgn}\left(x_{2}^{\prime}\right)\right] \frac{1}{\mu}
\end{array}\right.
$$

where $\mathrm{H}$ is a Heaviside function presenting the impact force in dimensionless form: 


$$
H=\left\{\begin{array}{l}
\beta \cdot\left(\left|x_{1}-x_{2}\right|-\gamma\right),\left(\left|x_{1}-x_{2}\right|-\gamma\right)>0 \\
0,\left(\left|x_{1}-x_{2}\right|-\gamma\right) \leq 0
\end{array}\right.
$$

The model in the dimensionless form described in Eq. (7) will be used to examine the dynamic response as well as to evaluate the system performance.

\section{PERFORMANCE AND DYNAMIC RESPONSE OF THE SYSTEM}

In this section, numerical solution of the mathematical model will be used to evaluate the system performance as well as the system dynamics. There are two major issues of the performance for this kind of locomotion system: the progression rate and the control strategy to obtain the expected direction of motion. The progression rate, hereafter is noted as $P$, is calculated from the displacement of the system frame, $x_{2}$ for the dimensionless time of 50 units. Similar previous studies usually evaluate effects of the investigated parameters by changing one parameter at a time. Here, we carried out the simultaneous effect of two parameters at a time instead. Two different working situations will be evaluated, including isotropic and anisotropic frictions.

The dynamic response of the system will be analysed by using bifurcation diagrams. Typically, a bifurcation diagram shows the values visited or approached asymptotically of a system as a function of a bifurcation parameter. In this study, Poincaré map of the relative velocity of the two massed was chosen as the values visited of the system. The excitation frequency, excitation amplitude, and mass ratio were selected as bifurcation parameters. The plotting process is described as below. For each value of the bifurcation parameter, calculate values of the Poincaré section of the relative velocity, $v_{1}-v_{2}$. The calculations were run for 300 cycles of the excited force. The first 100 cycles were considered to be transient and thus were omitted. The steady state of the next 200 points of the Poncare map was collected and plotted on the diagram.

\section{Performance and Dynamic Response Under Isotropic Friction Condition}

The isotropic friction is set with the same values of friction coefficient for both backward and forward motions, i.e. $\rho=1$. For a certain system, a better progression rate can be achieved by controlling either the excitation frequency, or excitation amplitude, or both of them. In order to highlight advantages of the proposed model, its performance and dynamic response will be compared with those of the similar system excited by squared waveform, using the same parameters.

Figure 3 shows contour plots of progression rates, $P$ obtained for two models working with the same set of parameters: the previous model with square excitation (figure $3 \mathrm{a}$ ) and the new model with half-sine excitation (figure $3 \mathrm{~b}$ ). As can be seen in the Figure, both backward (blue area) and forward (other colors) motions of the system can be observed. For the previous model, the highest progression as large as 14.15 , corresponding to frequency $\omega=2.09$ and the force ratio $\alpha=4.18$. The highest progression in the range of from 10 to 15 appeared in a small area (in red color in figure $3 a$ ). For the new model (figure 3b), the highest progression is of $21.04 \omega=2.98$ and $\alpha=2.73$. It is clearly that the highest of the progression of the new model (21.04) is much higher than that of the old model (14.15). Also, the red color for the highest range of progression (from 15 to 20) of the new model is much larger than that of the previous model, meaning that it is easier to control the new model to get the high progression than that of the old model. 
(a)

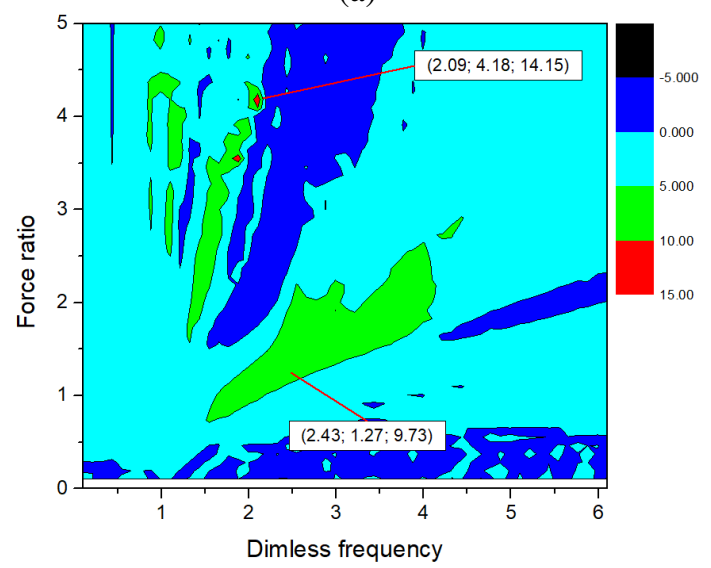

(b)

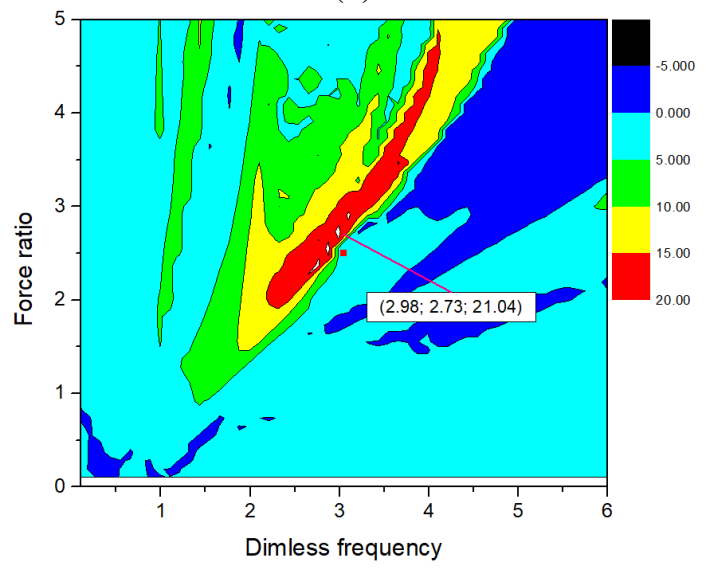

Figure 3: (Color Figure Online) Contour Map of Progression Rate, $P$ with Respect to Frequency of Excitation, $\omega$ and Force Ratio, $\alpha$, of the Model with (a) Squared Impulse Excitation and (b) Half-Sine Excitation; the Results Obtained for $\beta=46, \zeta=0.1, \gamma=0.58, \mu=1$, and $\rho=1$.

\section{Concurrent Effect of the Mass Ratio and the Excitation Frequency}

In the design stage, it should be considered that the masses and the excitation frequency have simultaneous and significant effects on the system performance (V.-D. Nguyen, Ho, et al., 2017), (V.-D. Nguyen, Duong, et al., 2017). Figure 4 illustrates the contour map of progression $P$, with respect to the frequency, $\omega$ and the mass ratio, $\mu$. As can be seen in figure 4, the peak areas (in yellow color) where the progression is high, of each model appeared at a certain range of frequency for a wide range of the mass ratio. With square excitation, the system can obtain highest frequency at frequency around 2 , and the mass ratio from 0.5 to 3 . With the half-sine excitation, the frequency range around 1.5 would provide high progression, and the mass ratio from 0.5 to around 3 . This observation is similar to results obtained in previous study (Huda \& Yu, 2015), (V.-D. Nguyen, Ho, et al., 2017), (V.-D. Nguyen, Duong, et al., 2017).

(a)

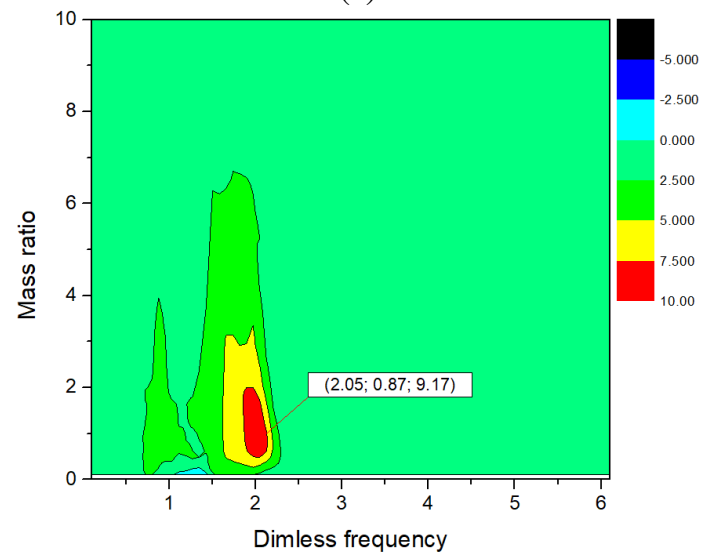

(b)

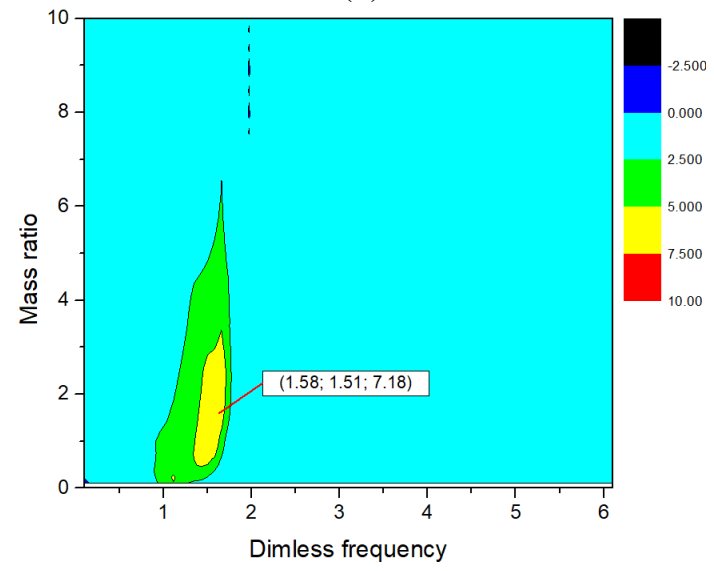

Figure 4: (Color Figure Online) Contour Map of Progression Rate, $P$ with Respect to Frequency of Excitation, $\omega$ and Mass Ratio, $\mu$, of the Model with (a) Squared Impulse Excitation and (b) Half-Sine Excitation; the Results Obtained for $\alpha=1, \beta=46, \zeta=0.1, \gamma=0.58, \mu=1$, and $\rho=1$.

\section{The Ability of Controlling the Motion Direction}

In order to check if the system can reverse the moving direction when reversing the excitation force, the progression rate is 
evaluated as function of the force ratio $\alpha$. The change of the sign of $\alpha$ from minus to plus means that the direction of the system motion changed from backward to forward ones.

Figure 5 ( $\mathrm{a}$ and $\mathrm{c}$ ) shows the changes of the progression when $\alpha$ varied from -3 to 3 . In order to gain insight into the dynamics response of the system changes, two corresponding bifurcation diagrams for varying force ratio in the same range are presented in figure $5 \mathrm{~b}$ and figure $5 \mathrm{~d}$.

In overall, it can be observed from the figure that all sub-plots have a symmetrical form. For opposite values of the excited force, each diagram reveres its waveform. The progression as well as the system behaviours for positive amplitudes are exactly as the same as those for negative amplitudes but in opposite values. In other words, reversing the direction of excitation force leads to reverse the moving direction of the system. Positive excitation provides forward motion and vise versa. Regarding the highest progressions obtained, the two kinds of excitation provided similar progression rate. However, with the half-sine excitation (figure $5 \mathrm{c}$ ), the system can move with significant rate ( $P>3$ for example) at a wider range of force $(|\alpha| \in[0.8,3])$, compared to that with square excitation $(|\alpha| \in[1,2.4]$ - see figure 5a).
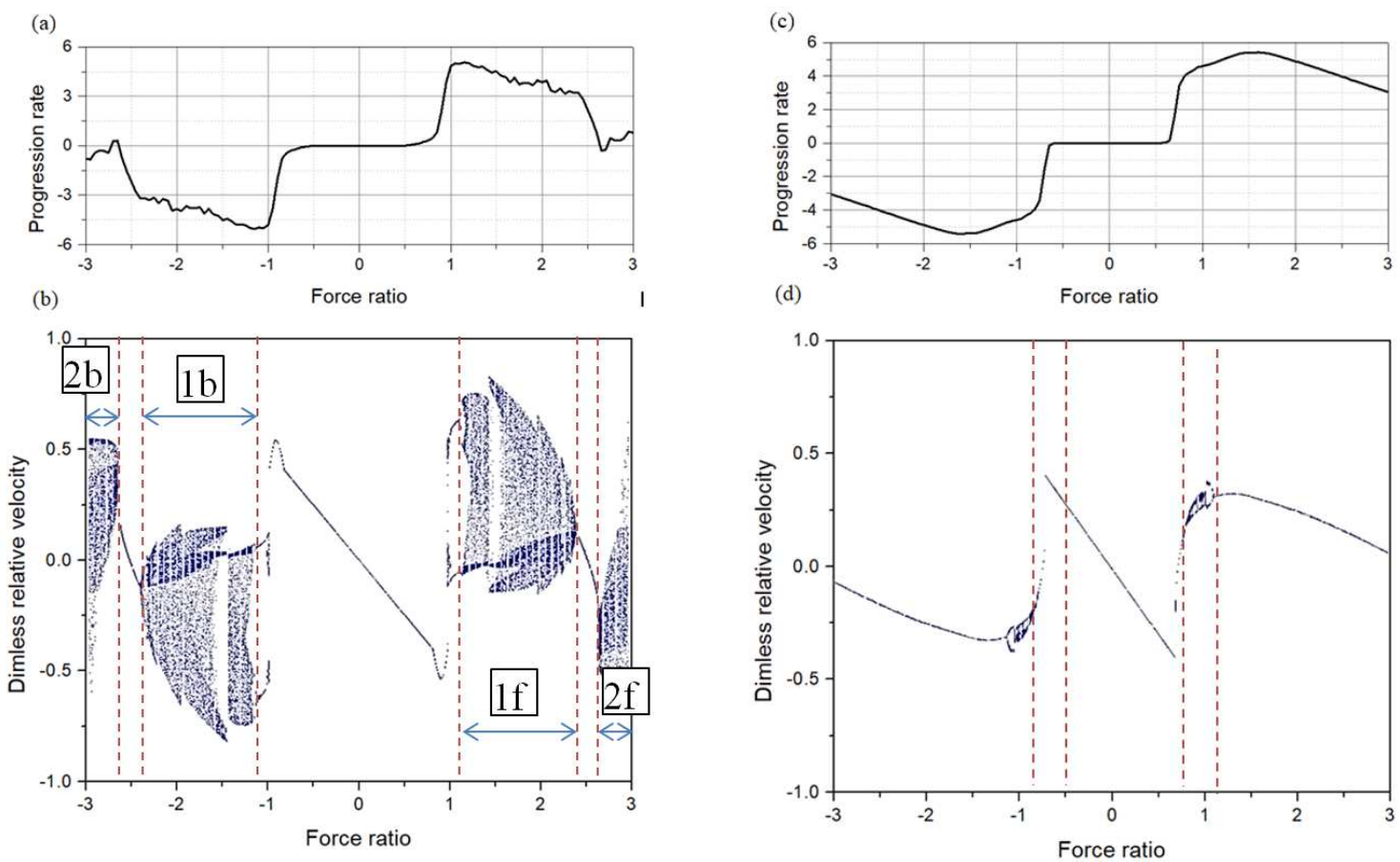

Figure 5: (a, c) Progression of the Model with (a) Impulse Force, (b) Half-Sine Force, with Respect to Force Ratio; (b, d) Bifurcation Diagram of the Relative Velocity v1-v2 for Varying Force Ratio; the Results Obtained for $\alpha=1, \beta=46, \zeta=0.1, \gamma=0.58, \mu=1$, and $\rho=1$.

Regarding the dynamics response, it can be seen in figure $5 \mathrm{~b}$ that chaotic responses appeared at four large ranges of the excited force with square waveform (Areas numbered $2 \mathrm{~b}, 1 \mathrm{~b}$ for backward motion and areas $1 \mathrm{f}$, 2f for forward motion in Figure 5b, corresponding to $|\alpha| \in[1.2,2.4]$ and $|\alpha|>2.6)$. In contrast, chaotic motion only appeared in small ranges of the force with half-sine form (figure $5 b:|\alpha| \in[0.8,1.2]$ ). It can be said that, in the investigated range of the force ratio, the half-sine excitation provided a more stable dynamics response than the square excitation did. 
(a)

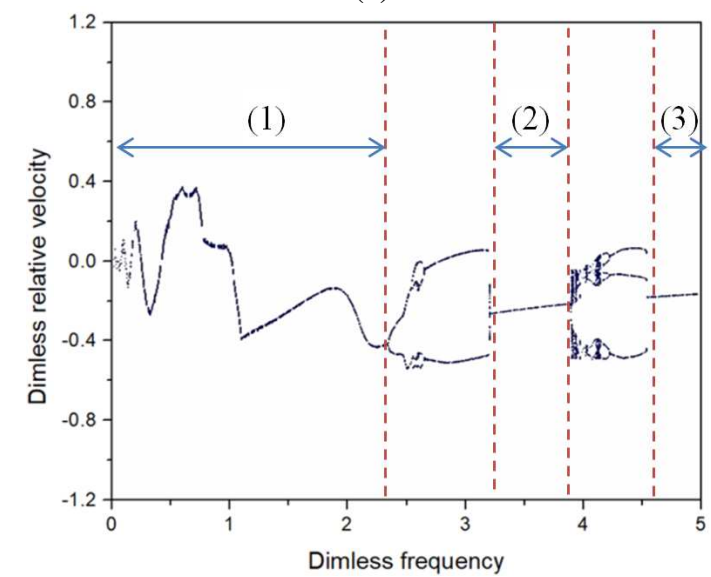

(b)

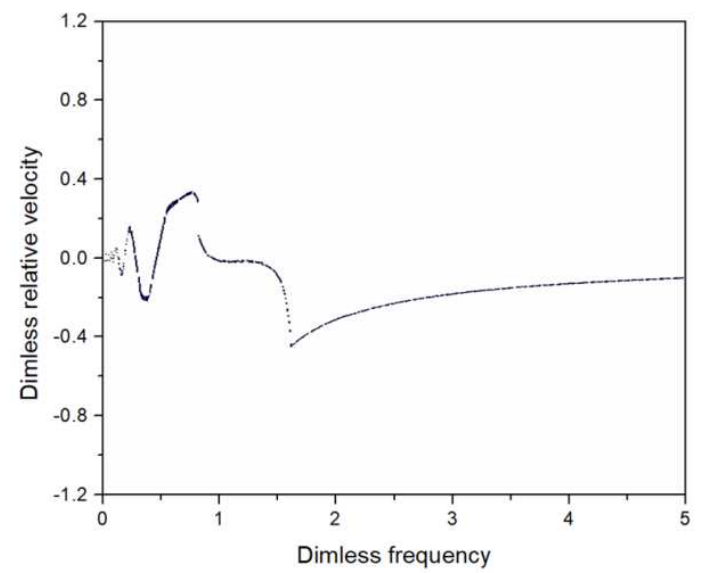

Figure 6: Bifurcation Diagram of the Relative Velocity $v 1-v 2$ for Varying Frequency of (a) the Previous Model and (b) the Current Model; the Results Obtained for $\alpha=1, \beta=46, \zeta=0.1, \gamma=0.58, \mu=1$, and $\rho=1$.

\section{Dynamic Response of the System}

To further validate and confirm if the new proposed model, with half-sine excitation, has a more stable dynamic response compared to the recent model, excited by square force, three couples of bifurcation diagrams were built and shown in figure 6, 7 and 8. In these cases, the excitation frequency was used as branching parameter. Three diagrams were made for different values of the force amplitude, $\alpha$ and the mass ratio, $\mu$.

In figure $6 \mathrm{a}$, the previous model has a moderately complex dynamics response. It only has period-one motion for three ranges (marked as numbers $1-3$ in figure $6 a$ ), including $\omega \in[0,2.3] ; \omega \in[3.25,3.85]$; and $\omega \in[4.6,5]$. Period-two and period-four motion appeared in the range $\omega \in[2.3,3.25]$. Chaotic response appeared in the range $\omega \in[3.85,4.6]$. On the other hand, the proposed model (Figure 6b) has period-one motion for all investigated values of the excitation frequency.

Similar phenomena can be seen in figure 7, where the mass ratio was set to be three, the highest ratio that can provide fast progressions (see section 3.1.2). The system working with square excitation has more complex dynamics response, as shown in figure $7 \mathrm{a}$. In contrast, the system excited by the half-sine force (figure $7 \mathrm{~b}$ ) has mostly period-one motion, except a small range $(\omega \in[1.4,1.6])$ that has a motion of period-two).

(a)

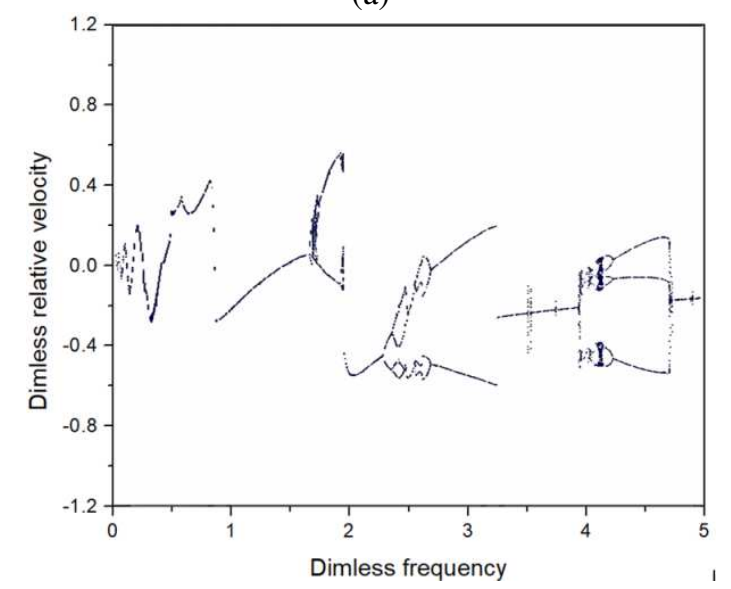

(b)

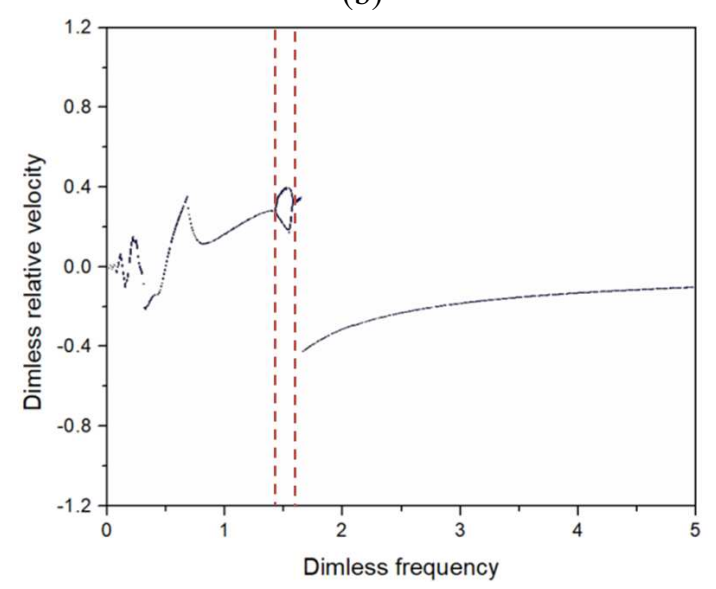

Figure 7: Bifurcation Diagram of the Relative Velocity v1-v2 for Varying Frequency of (a) the Previous Model and (b) the Current Model; the Results Obtained for $\alpha=1, \beta=46, \zeta=0.1, \gamma=0.58, \mu=3$, and $\rho=1$. 
The system behaviour at higher amplitude of excitation was also examined. Figure 8 presents bifurcation diagrams of the system with the force ratio $\alpha=1$. In overall, it can be seen that the system with square excitation (figure $8 \mathrm{a}$ ) has more complex dynamic characteristics, with three large ranges of frequency of chaotic, as numbered as (1), (2), and (3) in the figure. However, the system with half-sine excitation has only one range of chaotic motion (area (1)), as shown in figure $8 b$.

It can be noted that, the system with square excitation has more complex dynamic response than the system with half-sine excitation. The major reason can be explained as below.

(a)

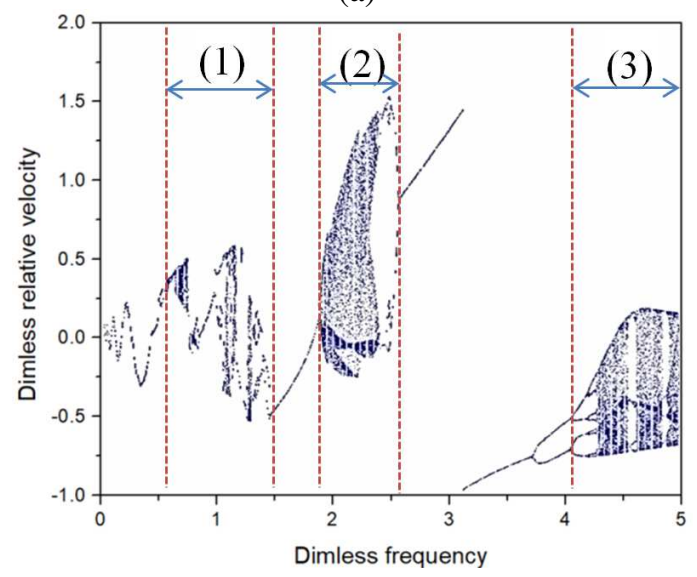

(b)

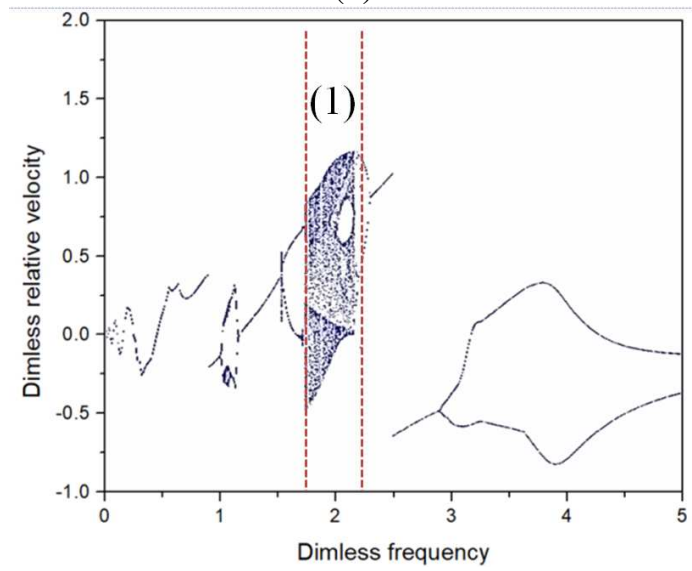

Figure 8: Bifurcation Diagram of the Relative Velocity $v 1-v 2$ for Varying Frequency of (a) the Previous Model and (b) the Current Model; the Results Obtained for $\alpha=2, \beta=46, \zeta=0.1, \gamma=0.58, \mu=3$, and $\rho=1$.

Looking at the time history of the relative motion of the internal mass and of the excitation force, as shown in figure 9, the square excitation remain its maximum value during a half of its period. In addition, the values just suddenly jumped up, remained and then abruptly jumped down. In contrast, the half-sine force just reached its peak and then immediately but smoothly went down.

On the one side, for the square excitation (figure 9a), after the mass reached its peak, it would tended to move back as results of the reaction of impact force and of the spring force. However, the excitation force still kept remained and thus did not permit the mass to turn back. The mass moved forward to hit the stop again, leading to two peaks of motion appeared. On the other side, the smooth peaks of sinusoidal form of the half-sine excitation had a better harmony with the natural motion of the mass after colliding with the stop (see figure $9 \mathrm{~b}$ ). 
(a)

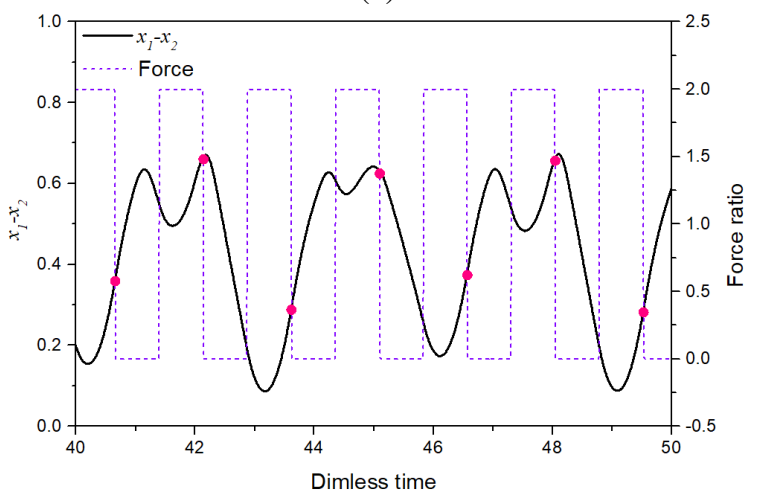

(b)

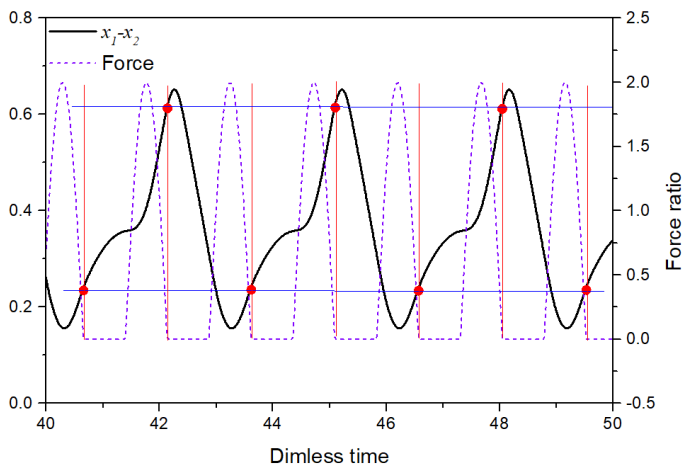

Figure 9: Time History of Relative Motion and Excitation Force of (a) the Previous Model and (b) the Current Model; the Results Obtained for $\alpha=2, \beta=46, \zeta=0.1, \gamma=0.58, \mu=3$, and $\rho=1$.

In figure 9, value of the relative motion of the mass at the instant when the force downed to zero was marked as red points (The Poincaré section). As can be seen in the figure, the Poincare values of the first case (figure 9a) were fluctuated among 5 levels, whereas there were only two levels at the later case (figure 9b). Compared to the bifurcation diagrams in figure 8 , these observations were well agreed with period-5 (figure $8 \mathrm{a}$ ) and period-2 (figure $8 \mathrm{~b}$ ).

\section{Working Ability Under Anisotropic Friction Condition}

The anisotropic friction occurred when the friction coefficients for backward and forward motion are different. In some previous studies (Xu \& Fang, 2019), (Du et al., 2018), (Zhan et al., 2018), the moving forward ability under anisotropic condition was evaluated, but only for a special case where the friction in forward direction is smaller than that in backward direction.

In this study, the friction condition was set by changing the value of the friction ratio. The friction ratio, $\rho$ is the scale of forward friction coefficient divided by backward friction coefficient. If $\rho<1$, the forward friction is smaller than the backward friction, and so on.

Figure 10 presents progression of the system obtained for different levels of the friction ratio.

(a)

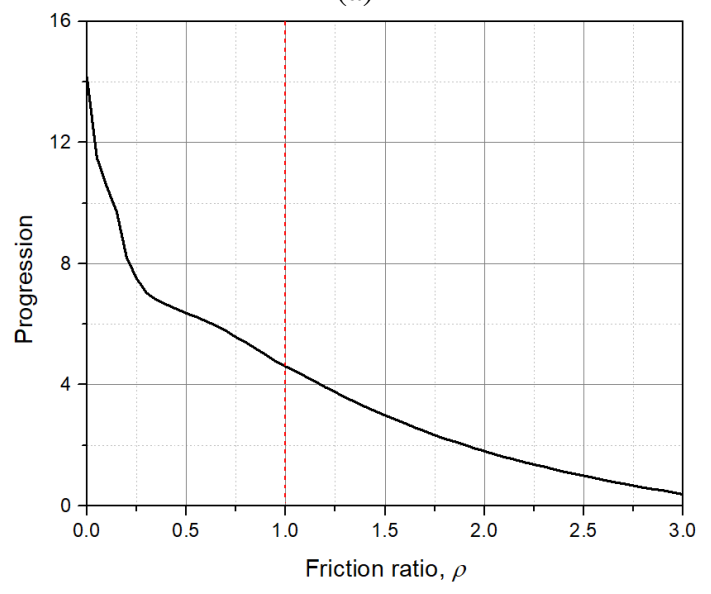

(b)

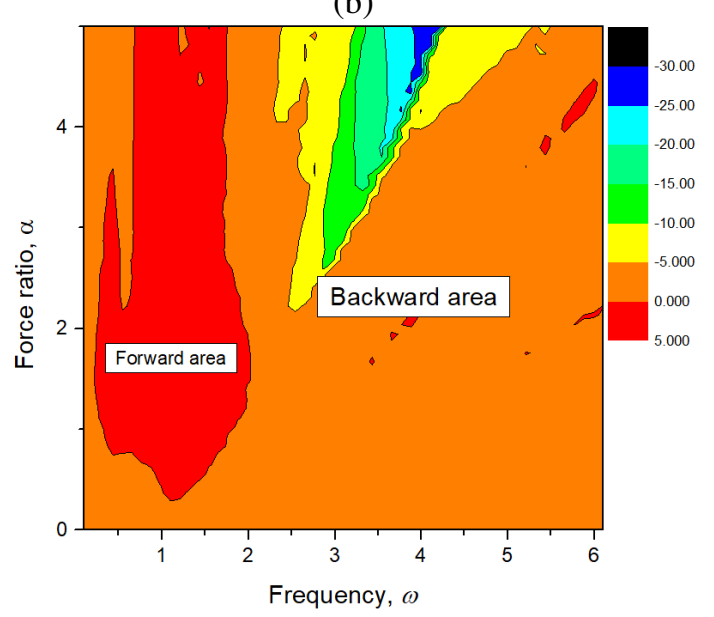

Figure 10: Moving Ability under Anisotropic Friction Condition: (a) Progression Rate for Varying Friction Ratio, with $\alpha=1, \beta=46, \zeta=0.1, \gamma=0.58, \mu=3$; (b) Progression Rate with Respect to Frequency and Force Ratio, with $\rho=2, \beta=46, \zeta=0.1, \gamma=0.58, \mu=3$. 
Figure 10a shows the progression obtained when the friction ratio changed from zero to three. In overall, increasing in the friction ratio resulted in decreasing the progression. It is reasonable as increasing the friction ratio mean that the forward friction becomes larger than backward friction, leading to an increase of the resistant force for forward motion. It also can be observed that, the system is able to move forward even when the forward friction force is three times higher than the backward friction force $(\rho=3)$. This would be a significant advantage of the proposed system.

To check with the moving forward ability with high value of forward friction, the progression rate for $\rho=2$ was plotted as a function of the excitation frequency and the force ratio in figure $10 \mathrm{~b}$. As can be seen in the figure, the system was still able to move forward for a larger range of the two parameters, as illustrated in the red area. All values of the excitation frequency larger than two would lead to backward movement, although the force acts on forward direction.

\section{CONCLUSIONS}

For vibration-driven autogenous mobile systems where both forward and backward progressions are desired, a careful design and/or a proper selection of oscillation parameters are still required. The system proposed in this paper can address such issues. The expected direction of system moving can be easily achieved by applying the excitation force in the same direction. A mathematical model was developed to describe the motions of the whole system under different friction conditions, including isotropic and anisotropic friction forces. The numerical results showed that the proposed system could provide faster moving rate with a larger range of parameters, compared to a similar model excited by square force. Using bifurcation analysis technique, it was found that the proposed system has simpler and more stable dynamics responses than the former did. The main reason behind this was explained initially. Further study would be recommended to explain this advantage of the new model. Moreover, the new system is strongly able to move in any desired direction, even the friction in that direction is higher than the friction in opposite direction.

\section{ACKNOWLEDGEMENTS}

This research was funded by Vietnam Ministry of Education and Training, under the grant number B2019-TNA-04.

The authors would like to express their gratitude to Thai Nguyen University of Technology, Thai Nguyen University, and Vinh University of Technology Education for rendering their support to the study.

\section{REFERENCES}

1. Cazalilla, J., Vallés, M., Valera, Á., Mata, V., \& Díaz-Rodríguez, M. (2016). Hybrid forcelposition control for a 3-DOF 1T2R parallel robot: Implementation, simulations and experiments. Mechanics Based Design of Structures and Machines, 44(1-2), 16-31. doi: 10.1080/15397734.2015.1030679

2. Chernous'ko, F. L. (2002). The optimum rectilinear motion of a two-mass system. Journal of Applied Mathematics and Mechanics, 66(1), 1-7. doi: https://doi.org/10.1016/S0021-8928(02)00002-3

3. Chernousko, F. L. (2018). Optimal Control of Two-Dimensional Motions of a Body by a Movable Mass. IFAC-PapersOnLine, 51(2), 232-235. doi: https://doi.org/10.1016/j.ifacol.2018.03.040

4. Du, Z., Fang, H., Zhan, X., \& Xu, J. (2018). Experiments on vibration-driven stick-slip locomotion: A sliding bifurcation perspective. Mechanical Systems and Signal Processing, 105, 261-275. doi: https://doi.org/10.1016/j.ymssp.2017.12.001

5. Duong, T.-H., Nguyen, V.-D., Nguyen, H.-C., Vu, N.-P., Ngo, N.-K., \& Nguyen, V.-T. (2018). A new design for bidirectional autogenous mobile systems with two-side drifting impact oscillator. International Journal of Mechanical Sciences, 140, 325- 
338. doi: 10.1016/j.ijmecsci.2018.01.003

6. Fan, J., Li, C., Yang, Z., Chen, S., Cao, J., \& Dou, C. (2020). On discontinuous dynamics of a 2-DOF oscillator with an onesided rigid obstacle. International Journal of Non-Linear Mechanics, 118, $103261 . \quad$ doi: https://doi.org/10.1016/j.ijnonlinmec.2019.103261

7. Golitsyna, M. V. (2018). Periodic Regime of Motion of a Vibratory Robot under a Control Constraint. Mechanics of Solids, 53(1), 49-59. doi: 10.3103/S002565441803007X

8. Grankin, A. N., \& Yatsun, S. F. (2009). Investigation of vibroimpact regimes of motion of a mobile microrobot with electromagnetic drive. Journal of Computer and Systems Sciences International, 48(1), 155-163. doi: $10.1134 / S 1064230709010158$

9. Gu, X. D., \& Deng, Z. C. (2018). Dynamical analysis of vibro-impact capsule system with Hertzian contact model and random perturbation excitations. Nonlinear Dynamics, 92(4), 1781-1789. doi: 10.1007/s11071-018-4161-x

10. Huda, M. N., \& Yu, H. (2015). Trajectory tracking control of an underactuated capsubot. Autonomous Robots, 39(2), $183-198$. doi: $10.1007 /$ s10514-015-9434-3

11. Li, H., Furuta, K., \& Chernousko, F. L. (2006, 13-15 Dec. 2006). Motion Generation of the Capsubot Using Internal Force and Static Friction. Paper presented at the Proceedings of the 45th IEEE Conference on Decision and Control.

12. Liu, P., Yu, H., \& Cang, S. (2018). Optimized adaptive tracking control for an underactuated vibro-driven capsule system. Nonlinear Dynamics, 94(3), 1803-1817. doi: 10.1007/s11071-018-4458-9

13. Liu, P., Yu, H., \& Cang, S. (2019). Modelling and analysis of dynamic frictional interactions of vibro-driven capsule systems with viscoelastic property. European Journal of Mechanics - A/Solids, 74, 16-25. doi: https://doi.org/10.1016/j.euromechsol.2018.10.016

14. Liu, Y., Islam, S., Pavlovskaia, E., \& Wiercigroch, M. (2016). Optimization of the Vibro-Impact Capsule System. Strojniški vestnik - Journal of Mechanical Engineering, 62(7-8), 430-439. doi: 10.5545/sv-jme.2016.3754

15. Liu, Y., Pavlovskaia, E., Hendry, D., \& Wiercigroch, M. (2013). Vibro-impact responses of capsule system with various friction models. International Journal of Mechanical Sciences, 72, 39-54. doi: 10.1016/j.ijmecsci.2013.03.009

16. Liu, Y., Pavlovskaia, E., \& Wiercigroch, M. (2015). Experimental verification of the vibro-impact capsule model. Nonlinear Dynamics, 83(1-2), 1029-1041. doi: 10.1007/s11071-015-2385-6

17. Liu, Y., Pavlovskaia, E., Wiercigroch, M., \& Peng, Z. (2015). Forward and backward motion control of a vibro-impact capsule system. International Journal of Non-Linear Mechanics, 70, 30-46. doi: 10.1016/j.ijnonlinmec.2014.10.009

18. Liu, Y., Wiercigroch, M., Pavlovskaia, E., \& Yu, H. (2013). Modelling of a vibro-impact capsule system. International Journal of Mechanical Sciences, 66, 2-11. doi: 10.1016/j.ijmecsci.2012.09.012

19. Mohamed, K., Elgamal, H., \& Elsharkawy, A. (2018). Dynamic analysis with optimum trajectory planning of multiple degreeof-freedom surgical micro-robot. Alexandria Engineering Journal, 57(4), 4103-4112. doi: https://doi.org/10.1016/j.aej.2018.10.011

20. Nguyen, V.-D., Duong, T.-H., Chu, N.-H., \& Ngo, Q.-H. (2017). The effect of inertial mass and excitation frequency on a Duffing vibro-impact drifting system. International Journal of Mechanical Sciences, 124-125, 9-21. doi: 10.1016/j.ijmecsci.2017.02.023

21. Nguyen, V.-D., Ho, H.-D., Duong, T.-H., Chu, N.-H., \& Ngo, Q.-H. (2017). Identification of the Effective Control Parameter to Enhance the Progression Rate of Vibro-Impact Devices With Drift. Journal of Vibration and Acoustics, 140(1), 011001. doi: 
$10.1115 / 1.4037214$

22. Nguyen, V.-D., Nguyen, H.-C., Ngo, N.-K., \& La, N.-T. (2017). A New Design of Horizontal Electro-Vibro-Impact Devices. Journal of Computational and Nonlinear Dynamics, 12(6). doi: 10.1115/1.4035933

23. Nguyen, V.-D., Woo, K.-C., \& Pavlovskaia, E. (2008). Experimental study and mathematical modelling of a new of vibroimpact moling device. International Journal of Non-Linear Mechanics, 43(6), 542-550. doi: 10.1016/j.ijnonlinmec.2007.10.003

24. Nguyen, V. D., \& Woo, K. C. (2008). New electro-vibroimpact system. Proceedings of the Institution of Mechanical Engineers, Part C: Journal of Mechanical Engineering Science, 222(4), 629-642. doi: 10.1243/09544062jmes833

25. Nikolay Bolotnik, F. C., Tatiana Figurina. (2015). Optimal Control of a Two-body Vibration-driven Locomotion System in a Resistive Environment. IFAC-PapersOnLine, 091-096.

26. Nunuparov, A., Becker, F., Bolotnik, N., Zeidis, I., \& Zimmermann, K. (2019). Dynamics and motion control of a capsule robot with an opposing spring. Archive of Applied Mechanics. doi: 10.1007/s00419-019-01571-8

27. Pavlovskaia, E., Wiercigroch, M., \& Grebogi, C. (2001). Modeling of an impact system with a drift. Phys Rev E Stat Nonlin Soft Matter Phys, 64(5 Pt 2), 056224. doi: 10.1103/PhysRevE.64.056224

28. Pavlovskaia, E., Wiercigroch, M., Woo, K.-C., \& Rodger, A. A. (2003). Modelling of Ground Moling Dynamics by an Impact Oscillator with a Frictional Slider. Meccanica, 38(1), 85-97. doi: 10.1023/a:1022023502199

29. Sapronov, K. A., Cherepanov, A. A., \& Yatsun, S. F. (2010). Investigation of motion of a mobile two-mass vibration-driven system. Journal of Computer and Systems Sciences International, 49(1), 144-151. doi: 10.1134/S1064230710010156

30. Shukla, A., \& Karki, H. (2016). Application of robotics in onshore oil and gas industry-A review Part I. Robotics and Autonomous Systems, 75, 490-507. doi: 10.1016/j.robot.2015.09.012

31. Sobolev, N. A., \& Sorokin, K. S. (2007). Experimental investigation of a model of a vibration-driven robot with rotating masses. Journal of Computer and Systems Sciences International, 46(5), 826-835. doi: 10.1134/s1064230707050140

32. Su, G., Zhang, C., Tan, R., \& Li, H. (2009, 10-15 Oct. 2009). A Design of the Electromagnetic Driver for the Internal ForceStatic Friction Capsubot. Paper presented at the 2009 IEEE/RSJ International Conference on Intelligent Robots and Systems.

33. Xu, J., \& Fang, H. (2019). Improving performance: recent progress on vibration-driven locomotion systems. Nonlinear Dynamics. doi: 10.1007/s11071-019-04982-y

34. Yaguchi, H., Kamata, K., \& Sugawara, H. (2016). Magnetic Actuator with Multiple Vibration Components Arranged at Eccentric Positions for Use in Complex Piping. Actuators, 5, 19. doi: 10.3390/act5030019

35. Yan, Y., Liu, Y., Manfredi, L., \& Prasad, S. (2019). Modelling of a vibro-impact self-propelled capsule in the small intestine. Nonlinear Dynamics, 96(1), 123-144. doi: 10.1007/s11071-019-04779-z

36. Yan, Y., Liu, Y., Páez Chávez, J., Zonta, F., \& Yusupov, A. (2018). Proof-of-concept prototype development of the selfpropelled capsule system for pipeline inspection. Meccanica, 53(8), 1997-2012. doi: 10.1007/s11012-017-0801-3

37. Zhan, X., Xu, J., \& Fang, H. (2018). A vibration-driven planar locomotion robot-Shell. Robotica, 36(9), 1402-1420. doi: $10.1017 /$ S0263574718000383 